\title{
High-field Zeeman contribution to the trion binding energy
}

\author{
T. Vanhoucke* and M. Hayne \\ Laboratorium voor Vaste-Stoffysica en Magnetisme, K. U. Leuven, Celestijnenlaan 200 D, B-3001 Leuven, Belgium \\ M. Henini \\ School of Physics and Astronomy, University of Nottingham, Nottingham NG7 2RD, United Kingdom \\ V. V. Moshchalkov \\ Laboratorium voor Vaste-Stoffysica en Magnetisme, K. U. Leuven, Celestijnenlaan 200 D, B-3001 Leuven, Belgium
}

(Received 24 October 2001; published 8 January 2002)

\begin{abstract}
We examine the role of the Zeeman interaction in determining the bound states of the trion at magnetic fields up to $50 \mathrm{~T}$. Polarization-sensitive photoluminescence measurements on the singlet state of the positively charged trion $\left(\mathrm{X}^{+}\right)$in GaAs quantum wells demonstrate a $60 \%$ enhancement of the $g$ factor compared to that of the neutral exciton $\left(X_{0}\right)$ in the same sample. This leads to a situation in very high fields where the Zeeman splitting of $X^{+}$is sufficiently large to determine whether a state is bound or not, and so calls for a reexamination of what is meant by the binding energy of few particle systems.
\end{abstract}

DOI: 10.1103/PhysRevB.65.041307

PACS number(s): 78.67.De, 71.35.Ji, 73.21.Fg, 78.55.Cr

Progress in semiconductor growth techniques has enabled the study of a rich diversity of phenomena in lowdimensional structures. ${ }^{1-4}$ Generally, the confinement of charge carriers in such systems dominates the physics, but spin can also play an important role. It was shown, for example, that the spin alignment of the electrons in a twodimensional electron gas determines the fractional quantum Hall ground state at Landau-level filling factor $\nu=2 / 3{ }^{2}$ while close to $\nu=1$, quasiparticle excitations called skyrmions are formed as a low-energy excitation of a purely spin origin. ${ }^{3}$ The electrical transport through quantum dots has provided insight into the Kondo effect in low-dimensional systems, and this has affirmed the importance of the mutual spin alignment of the confined and free electrons. ${ }^{4}$ Here we discuss the role of spin in determining the binding energy $E_{b}^{*}$ of the excess charge carrier in quantum wells of the charged exciton, also called a trion. Charged excitons $X^{*}$ are formed when the electron-hole pair of the neutral exciton, $X_{0}$, binds a third charge carrier. A negatively charged exciton $X^{-}$consists of one hole and two electrons, while a positively charged exciton $X^{+}$contains two holes and one electron. $X^{-}$ has been the subject of intense theoretical ${ }^{5}$ and experimental $^{6-12}$ investigation in recent years, while $X^{+}$has received little attention. ${ }^{11-14}$ Theoretically, this is probably due to the complexity of the valence band, while the difficulty of growing high-quality two-dimensional hole gases (2DHG) hampers the experimental investigation.

In this study we turn our attention to $X^{+}$because, as will be shown later, it has a larger Zeeman splitting compared to $X^{-}$. This makes it a convenient model to consider the role of the Zeeman interaction in the binding energy of few particle systems. The $X^{+}\left(X^{-}\right)$binding energy, $E_{b}^{+}\left(E_{b}^{-}\right)$, is defined as the energy needed to remove the "second" hole (electron). Since the first observation of $X^{-}$in $1993,{ }^{10}$ a number of groups studied $E_{b}^{-}$and good agreement between theory and experiment has finally been obtained. ${ }^{6}$ In contrast, $E_{b}^{+}$is much less investigated and its field dependence is essentially unknown. In this communication, we present polarizationsensitive photoluminescence (PL) experiments on $X^{+}$in magnetic fields $B$ up to $50 \mathrm{~T}$. Our observations of the $X_{0}$ and $X^{+}$effective $g$ factor, $g_{0}$ and $g^{+}$, respectively, ${ }^{15}$ leads us to conclude that the Zeeman interaction plays a crucial role in stabilizing the $X^{+}$in high fields and that the almost arbitrary way in which the Zeeman contribution to the $X^{*}$ binding energy is considered needs to be re-evaluated.

An examination of the literature ${ }^{5-8,11}$ reveals that the infield binding energy of the charged exciton is given according to two different schemes shown in Fig. 1. One scheme (scheme I in Fig. 1) is based on taking the difference in PL energy between $X_{0}\left(\sigma^{-}\right)$and $X^{*}\left(\sigma^{-}\right)$, where $\sigma^{+}$and $\sigma^{-}$ indicate the right- and left-handed circularly polarized light components, respectively, and gives the binding energy of the lower energy $X^{*}$ spin state of the singlet $(\uparrow \downarrow) \uparrow$. In the $X^{*}$ spin alignment notation of Fig. 1, the first two arrows indicate the mutual alignment of the identical particles while the last arrow shows the spin of the third charge carrier with

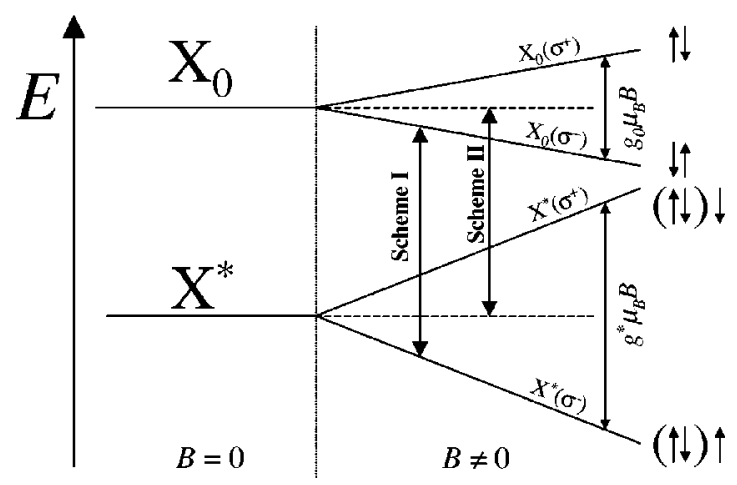

FIG. 1. Schematic illustration of how the trion binding energy is obtained including (scheme I) or excluding (scheme II) the Zeeman interaction. For $X_{0}$ only the optically active states are shown here, and for $X^{*}$ only the singlet state is shown, but similar approaches can be used for the triplet. The spin notation is explained in the text. 
$\uparrow$ and $\downarrow$ for spin up and down, respectively. For $X_{0}$, the first and second arrow indicate the electron and hole spin, respectively. The second scheme (scheme II in Fig. 1) is the subtraction of the mean Zeeman energies of $X_{0}$ and $X^{*}$. For both schemes a similar approach can be used for the triplet state of $X^{*}$. Since the PL intensity of the $\sigma^{+}$components is an order of magnitude lower than $\sigma^{-}$, and therefore often not experimentally observable, ${ }^{6}$ the former scheme is usually used in experiments. ${ }^{6,7}$ Conversely the latter scheme is mostly used in theoretical studies ${ }^{5}$ due to the complexity of including a full consideration of the Zeeman interaction. As can be seen from Fig. 1, consistency between the two schemes is obtained under the not unreasonable assumption that $g_{0}=g^{*}$, where $g^{*}$ is the $X^{*} g$ factor. Recent experiments on $X^{-}$have shown that $g_{0} \neq g^{-}\left(g^{-}\right.$is the $X^{-} g$ factor) though the difference is quite small, so there is no qualitative change in the $X^{-}$binding energy derived by the two schemes at experimentally obtainable magnetic fields. This is not the case for $X^{+}$, where, as we shall demonstrate, the large difference in $X_{0}$ and $X^{+} g$ factors gives rise to a qualitatively different prediction as to whether the singlet is bound in high fields.

The quantum well (QW) samples were grown on a (311)A GaAs substrate by molecular-beam epitaxy and modulation doped with $\mathrm{Si}$ as acceptor. The QW width was $150 \AA$ for all samples while the undoped spacer was 800,600 , and $200 \AA$ for samples $A, B$, and $C$, respectively. The experiments were performed at a temperature of 1.2 and $4.2 \mathrm{~K}$ with the magnetic field parallel to the sample growth direction, i.e., in the Faraday configuration. A $532 \mathrm{~nm}$ solid-state laser was used to excite the sample, while the PL light was collected by six optical fibers arranged symmetrically around the central excitation laser fiber and dispersed onto an intensified chargecoupled-device detector at a spectral resolution better than $0.3 \mathrm{meV}$. Above $\mathrm{Al}_{x} \mathrm{Ga}_{1-x} \mathrm{As}$ band-gap illumination is used to reduce the 2DHG density (optical depletion) without introducing further disorder. ${ }^{16}$ A $28 \mathrm{mF}$ capacitor bank at $<5 \mathrm{kV}$ was discharged into a nitrogen-cooled coil, giving magnetic fields up to $50 \mathrm{~T}$ with a $27 \mathrm{~ms}$ pulse duration. Using a field resolution of $\pm 1 \%$ we obtained a photon integration time of $2.2 \mathrm{~ms}$. An in situ polarizer in combination with reversing the magnetic-field direction enabled us to distinguish between the $\sigma^{+}$and $\sigma^{-}$PL components.

The field dependence of the PL energy of sample $A$ at 1.2 $\mathrm{K}$ is shown in Fig. 2 with the open and filled symbols indicating the $\sigma^{+}$and $\sigma^{-}$polarization, respectively. Samples $B$ and $C$ behave very similarly and are not shown here. Measurements at $4.2 \mathrm{~K}$ show no difference for all samples except for an even faster decrease of the PL intensity with magnetic field. This is consistent with Ponomarev et al. ${ }^{13}$ who studied samples from the same wafer at lower fields and in the absence of polarization sensitivity. They found that at zero field the high-energy PL peak intensity drops down dramatically above $2 \mathrm{~K}$, while the other PL peak gradually weakens above $3 \mathrm{~K}$. The low-energy PL peak at zero field is assigned to the singlet spin-state of $X^{+}$(circles in Fig. 2), and the highenergy peak is assigned to $X_{0}$ (triangles in Fig. 2). The assignment of the lines is motivated by a comparison of their relative intensities for all three samples under the same ex-

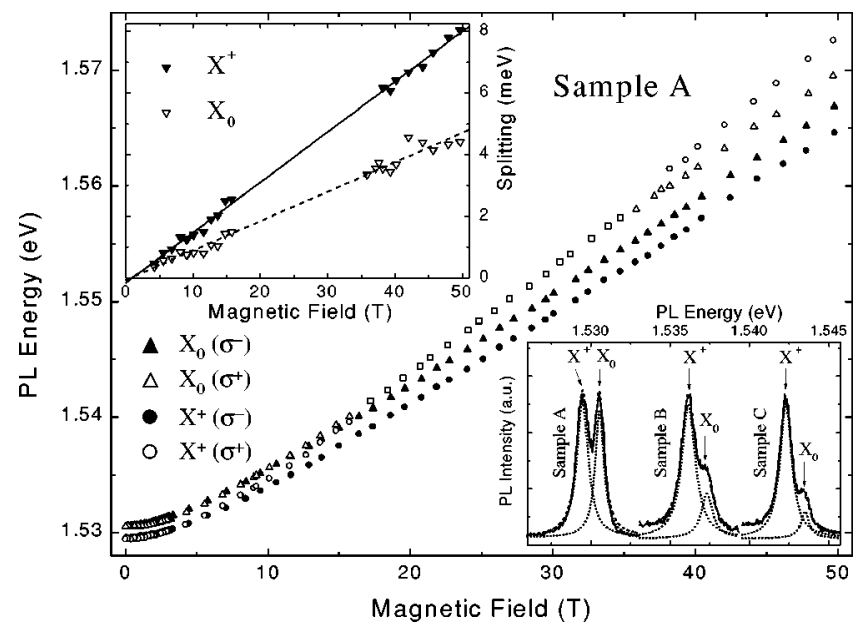

FIG. 2. Magnetic-field dependence of the PL energy of sample $A$ at $1.2 \mathrm{~K}$. The open and filled symbols represent the right- $\left(\sigma^{+}\right)$and left-handed $\left(\sigma^{-}\right)$circularly polarized PL, respectively. The upper inset shows the Zeeman splitting of $X^{+}$and $X_{0}$, while the lower inset displays zero-field spectra of samples $A$ to $C$ fitted with Lorentzian curves (see text for details).

perimental conditions (lower inset of Fig. 2). (Note that in the lower inset of Fig. 2 the PL spectra of samples $B$ and $C$ are shifted by $+6 \mathrm{meV}$ and $+12 \mathrm{meV}$, respectively, for clarity.) The relative intensities of the peaks are determined by Lorentzian fits and after subtracting a background for samples $B$ and $C$. The weakening of the $X_{0}$ peak relative to $X^{+}$with decreasing spacer width (going from sample $A$ to sample $C$ ) at zero field is a result of a higher 2DHG density, which is directly related to the spacer width by the hole tunneling time. ${ }^{16}$ This is in agreement with the data of Ponomarev et al. ${ }^{13}$ who show essentially the same spectra. At zero field, where both $\sigma^{+}$and $\sigma^{-}$polarizations coincide, the binding energy is simply given by the energy separation of the $X^{+}$and $X_{0}$ PL peaks and yields a value of $1.1 \mathrm{meV}$. Glasberg et al. ${ }^{11}$ have found the same value for a $200 \AA$ QW, while an $E_{b}^{+}$of $1.0 \mathrm{meV}$ was determined by Shields et al. ${ }^{14}$ for a $300 \AA$ QW. Measurements on a further sample with a $200 \AA \mathrm{QW}$, not discussed here, also give a binding energy of $1.0 \mathrm{meV}$. The proximity of all these values clearly indicates a lack of variation in $E_{b}^{+}$at zero field with quantum-well width.

In magnetic field both polarizations of $X_{0}$ and $X^{+}$are resolved, except between 16 and $35 \mathrm{~T}$ where $X_{0}\left(\sigma^{+}\right)$and $X^{+}\left(\sigma^{+}\right)$merge due to a small difference in PL energy. Above $35 \mathrm{~T}$ all peaks are resolved again, resulting in four different PL lines at $50 \mathrm{~T}$. Taking the difference between the $\sigma^{+}$and $\sigma^{-}$PL energies we observe a linear Zeeman splitting (upper inset of Fig. 2) for $X_{0}$ and $X^{+}$. No data are available between 16 and $35 \mathrm{~T}$ for the reason mentioned above. The same linear behavior, demonstrating a field independent $g$ factor, was found for $X^{-}$in previous experiments. ${ }^{6}$ The slope of the Zeeman splitting gives $g_{0}=1.7$ which is slightly higher than the corresponding value of 1.5 for a $100 \AA$ $n$-doped QW sample under similar experimental conditions. ${ }^{6}$ The same data show an enhancement of the $X^{-} g$ factor to 1.9 in the same $100 \AA \mathrm{QW}$ sample. Here we find an $X^{+} g$ 


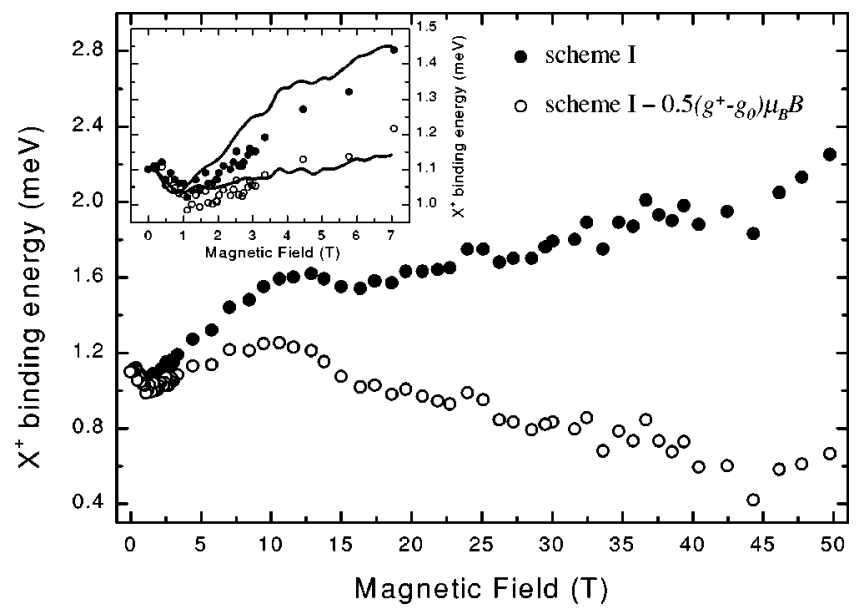

FIG. 3. Experimental results of the $X^{+}$binding energy obtained by using scheme I (filled circles), while the open circles represent the "corrected" $E_{b}^{+}$by subtracting $0.5\left(g^{+}-g_{0}\right) \mu_{B} B$. The "corrected" $E_{b}^{+}$indicates that $X^{+}$is likely to be unbound at $B>75 \mathrm{~T}$, but such an interpretation does not include the Zeeman interaction correctly. The inset compares our low-field data with those obtained by Glasberg et al. (Ref. 12) (solid lines).

factor of 2.8 , enhanced by more than $60 \%$ over the $X_{0}$ value. Glasberg et al. ${ }^{11}$ measured the neutral and charged exciton $g$ factors at fields up to $7 \mathrm{~T}$ in a special structure in which $X^{+}$ and $X^{-}$are observed in the same sample. Their data show a strong field dependence of $g^{+}$up to $4 \mathrm{~T}$, where it reaches a constant value of $-2 .{ }^{17}$ Such behavior is not inconsistent with our data since we cannot resolve the spin-splitting to such low fields. On the other hand, $\left|g^{-}\right|$and $\left|g_{0}\right|$ monotonically increase in their data, reaching nonsaturated values of 1 and 0.7 , respectively at $7 \mathrm{~T}$. A key point though, crucial to the problem we are considering here, i.e., the effect of the Zeeman interaction on the bound states of the charged exciton, is that Glasberg et al. ${ }^{11}$ also found $\left|g^{+}\right|>\left|g^{-}\right|>\left|g_{0}\right|$.

The filled symbols in Fig. 3 represent $E_{b}^{+}$determined from our data using scheme I of Fig. 1, while the open symbols show the "corrected" $E_{b}^{+}$by subtracting $0.5\left(g^{+}-g_{0}\right) \mu_{B} B$. This correction has been used rather than taking the difference in mean Zeeman energy since no reliable $\sigma^{+}$data are available between 16 and $35 \mathrm{~T}$, as mentioned above. The filled circles of Fig. 3 (scheme I) indicate a substantial increase of $E_{b}^{+}$from $1.1 \mathrm{meV}$ at zero field to 1.6 $\mathrm{meV}$ at $B=14 \mathrm{~T}$ while a further increase of the magnetic field slowly changes $E_{b}^{+}$to $2.3 \mathrm{meV}$ at $50 \mathrm{~T}$. In contrast, the corrected $E_{b}^{+}$remains almost flat at low fields and then gradually decreases with increasing field. The inset of Fig. 3 shows our low-field data together with the data from Glas- berg et al. ${ }^{11}$ for a $200 \AA$ QW sample. Both sets of data are in very good agreement, and even reproduce the small minimum in $E_{b}^{+}$around $1 \mathrm{~T}$. Recent theory on $X^{+}$also shows a similar low-field behavior for $E_{b}^{+} \cdot{ }^{18}$

It is clear from Figs. 1 and 3 that because $g_{0} \ll g^{+}$the two schemes result in a completely different magnetic-field behavior of $E_{b}^{+}$. Indeed, the binding energies in Fig. 3 diverge with increasing field such that if this trend were to continue to even higher fields, the Zeeman corrected binding energy would reach zero at $B>75 \mathrm{~T}$, meaning that $X^{+}$becomes unbound at very high fields. However, according to the data in Fig. 2, this is clearly not the case. The low-energy singlet spin-state $(\uparrow \downarrow) \uparrow$ of $X^{+}$remains the lowest energy state and no crossing between $X_{0}\left(\sigma^{-}\right)$and $X^{+}\left(\sigma^{-}\right)$is revealed. Indeed, the filled symbols in Fig. 3 indicate a saturation or even a slight increment of $E_{b}^{+}$with field. Thus, the singlet state remains bound at very high fields as a direct result of the Zeeman interaction. This means that the Zeeman splitting cannot be ignored when considering bound states of charged excitons. Indeed, the binding energy of a system is defined for the lowest energy state, and therefore one should strictly use scheme I and not scheme II anyway. ${ }^{19}$ As mentioned above, we note that the influence of the Zeeman interaction on the binding energy is directly related to the difference between $g_{0}$ and $g^{*}$. Since $g^{-}$is comparable with $g_{0},{ }^{6,11} E_{b}^{-}$ will not be influenced to a great extent if scheme II is used rather than scheme I, at experimentally accessible magnetic fields at least. This, combined with the assumption that $g^{*}$ $=g_{0}$, certainly explains why this problem has not been consistently addressed in the past. A detailed quantitative consideration of the magnetic-field dependence of $E_{b}$ for $X^{-}$ should, of course, also include the role of the Zeeman interaction.

We have presented PL data of $X^{+}$in high magnetic fields. We have shown that due to the relatively large $X^{+} g$ factor, it is an ideal system for demonstrating the importance of the Zeeman interaction in the binding energy of few particle systems. We have applied and analyzed two qualitatively different schemes for specifying the binding energy, and found that one can result in misleading conclusions as to whether a charged exciton state remains bound at high fields. Indeed, in the case of $X^{+}$the lower energy spin-singlet state remains bound at very high fields as a direct consequence of the Zeeman interaction.

This work was supported by the FWO-Vlaanderen, the Flemish GOA, the Belgian IUAP programs, the EPSRC (UK), the K.U. Leuven VIS 00/001 project, and Philips Optical Storage.
*Email: tony.vanhoucke@fys.kuleuven.ac.be

${ }^{1}$ A.D. Yoffe, Adv. Phys. 1, 1 (2001).

${ }^{2}$ T. Chakraborty, Adv. Phys. 49(8), 959 (2000).

${ }^{3}$ S.M. Girvin, Phys. Today 53(6), 39 (2000); S.E. Barrett, G. Dabbagh, L.N. Pfeiffer, K.W. West, and R. Tycko, Phys. Rev. Lett. 74, 5112 (1995).

${ }^{4}$ L. Kouwenhoven and L. Glazman, Phys. World January, 33
(2000).

${ }^{5}$ D.M. Whittaker and A.J. Shields, Phys. Rev. B 56, 15185 (1997);

A. Wójs, J.J. Quinn, and P. Hawrylak, ibid. 62, 4630 (2000);

C. Riva, F.M. Peeters, and K. Varga, ibid. 63, 115302 (2001).

${ }^{6}$ T. Vanhoucke, M. Hayne, M. Henini, and V.V. Moshchalkov, Phys. Rev. B 63, 125331 (2001). 
${ }^{7}$ A. J. Shields, M. Pepper, P. C. M. Christianen, J. C. Maan, M. Y. Simmons, and D. A. Ritchie, Proceedings of the 12th International Conference on High Magnetic Fields in the Physics of Semiconductors II Volume 2, Wurzburg, 1996 (World Scientific, Singapore, 1997), p. 737.

${ }^{8}$ M. Hayne, C.L. Jones, R. Bogaerts, C. Riva, A. Usher, F.M. Peeters, F. Herlach, V.V. Moshchalkov, and M. Henini, Phys. Rev. B 59, 2927 (1999); G. Yusa, H. Shtrikman, and I. BarJoseph, cond-mat/0103561 (unpublished).

${ }^{9}$ D. Sanvitto, R.A. Hogg, A.J. Shields, D.M. Whittaker, M.Y. Simmons, D.A. Ritchie, and M. Pepper, Phys. Rev. B 62, R13 294 (2000).

${ }^{10}$ K. Kheng, R.T. Cox, Y. Merle d'Aubigné, F. Bassani, K. Saminadayar, and S. Tatarenko, Phys. Rev. Lett. 71, 1752 (1993).

${ }^{11}$ S. Glasberg, G. Finkelstein, H. Shtrikman and I. Bar-Joseph, Phys. Rev. B 59, R10 425 (1999).

${ }^{12}$ G. Finkelstein, H. Shtrikman and I. Bar-Joseph, Phys. Rev. B 53, R1709 (1996).

${ }^{13}$ Y.V. Ponomarev, A. Usher, P.J. Rodgers, B.L. Gallagher, M. Henini, and G. Hill, Phys. Rev. B 54, 13891 (1996).
${ }^{14}$ A.J. Shields, J.L. Osborne, M.Y. Simmons, M. Pepper, and D.A. Ritchie, Phys. Rev. B 52, R5523 (1995).

${ }^{15}$ Here we consider the effective $g$ factor of the (charged) exciton, which includes the spin splitting of both initial and final states. Thus one might expect $g_{0}=g^{*}=g_{e}+3 g_{h}$, where $g_{e}$ and $g_{h}$ are the single particle electron and hole $g$ factors. See, for example, Ref. 6.

${ }^{16}$ M. Hayne, A. Usher, A.S. Plaut, and K. Ploog, Phys. Rev. B 50, 17208 (1994); Y.V. Ponomarev, A. Usher, M. Hayne, P.J. Rodgers, B.L. Gallagher, M. Henini, and G. Hill, Solid State Commun. 102, 53 (1997).

${ }^{17}$ In contrast with our findings, Glasberg et al. have observed $\sigma^{+}$to have the lowest PL energy. Therefore, the sign of the $g$ factor in Ref. 11 is opposite to ours.

${ }^{18}$ C. Riva, F.M. Peeters, and K. Varga, Phys. Rev. B 64, 235301 (2001).

${ }^{19}$ One can also define a binding energy for an excited state, for example the triplet, but in this case the same argument about use of scheme I applies. 